\title{
Development of multimedia animations - a contribution of informatics teaching to media studies
}

\author{
Michael Weigend \\ Fernuniversität Hagen, Holzkamp-Gesamtschule Witten, Germany
}

michael.weigend@fernuni-hagen.de

Key words: Curriculum changes, Informatics, Media Education, Problem Solving

\begin{abstract}
The development of a multimedia visual model can be an informative process that requires and encourages a large number of abilities. This paper focuses on three dimensions of the modelling process, all connected with certain abilities. Firstly, an informative model is a simplified image of a real system. In this respect modellers are required to observe reality well, to recognise and describe structures. Secondly, the model can be regarded as a medium through which certain mental constructs and contents can be imparted to recipients (communicative aspect). Finally, a multimedia model (especially if it is to be attractive) is a complex, technical product whose implementation demands the use of methods of information technology, for example object-oriented modelling. Using the example of the object-oriented modelling of a waterworks with the help of Flash 5, the paper discusses under what conditions the development of animation in IT teaching can help modelling abilities.
\end{abstract}

\section{MODEL FORMATION IN SCHOOL}

The development of models to explain real phenomena is very important, particularly in the teaching of science. There are detailed concrete teaching suggestions (see e.g. Ossimitz 2001) which are often based on the following pattern. On the basis of observations of a realistic, dynamic system (e.g. a forest ecosystem) a colloquial word model is formulated. This is then developed into a mathematical model and a computer simulation. In

The original version of this chapter was revised: The copyright line was incorrect. This has been corrected. The Erratum to this chapter is available at DOI: 10.1007/978-0-387-35663-1_34 
simulation runs with different input data the behaviour of the program is compared with the behaviour of the real system. If differences are discovered the real system is studied again in more detail and the model is changed. The model forming cycle continues until the behaviour of the model conforms well with the observed behaviour of the real system (Bossel 1987).

This process is supported by software tools such as Dynasys that allow the input of a model, a scenario of starting values and the output of a system's behaviour in a simulation. One weakness of existing systems is the limited demand on the creativity and imagination of the pupils. The possibilities in the definition of a model are basically limited to the arrangement of given symbols. The output during the simulation consists mainly of time and phase diagrams.

In the leisure market many successful simulation programs follow a common principle. Players build an artificial world from components (in SimCity they build a town with streets, shops, hospitals and industrial areas) which operates according to certain prescribed rules. The (sensory) attraction is in the observation of the effects of changes (e.g. the increase in tax yield because the town has highly successful industrial areas and has been able to draw in investors).

Programs of this kind are attractive and are used in many American schools as a medium for teaching pupils about complex systems such as social systems or ecological systems (Diener et al 1998). One disadvantage is that the logical model on which the system is based is not revealed to the player and cannot be changed.

As an alternative to the two above-mentioned approaches, this paper proposes the design of a visual model as multimedia interactive software (animation) with the help of Flash 5. In two respects we go beyond the modelling of dynamic systems suggested by Bossel. Firstly, questions of the communication design are included. The target product - the model - is seen as a medium conceived for a certain group of recipients. Secondly, in the model formation process, aspects of the technical realisability are taken into consideration.

An animation is a relatively complex system which can only be developed using information technology methodology. While there are many relevant informatic modelling techniques available (Hubwieser 1999), here we only consider the development of a class structure within the framework of object-oriented modelling. 


\section{ANALYSIS OF A WINDOW FROM REALITY - EXAMPLE: THE WITTEN WATERWORKS}

It is an advantage if the "window on reality" has the following features observability, structural feasibility and sufficient size. As an example, consider the modelling of a waterworks which was undertaken by pupils in years 9 to 11 at Holzkamp comprehensive school in Witten as part of a project week. (Detailed documentation, including the Flash animations mentioned in this paper are at http://www.informatik.fernunihagen.de/schulinformatik/projekt43/).

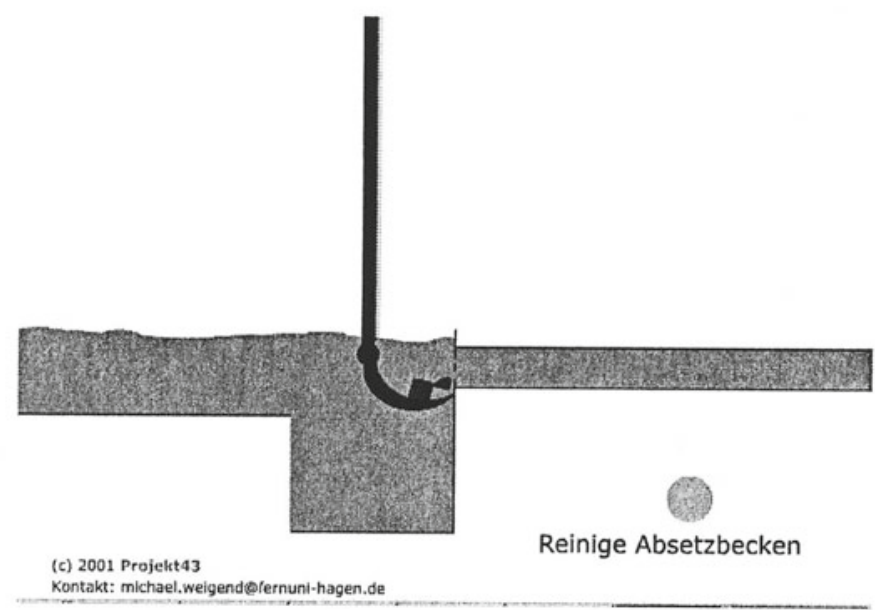

Figure 1. Flash animation screenshot of the fine screen system

The aim of the project was the creation of a multimedia animation with interactive, dynamic, visual and audio components - important components such as the fine screen which removes (strains) coarse particles from the river water and a settling pond that is occasionally cleaned by means of a mechanical gripper (Figure 1).

What makes a waterworks a suitable object for modelling?

- Observability. The window to be modelled is accessible to sensory perception and independent exploration. The waterworks can be visited. Its components can be photographed and measured. Original sounds of rushing water, pumps and compressed air can be recorded and incorporated into the animation. There are experts whom the pupils can question about details that are not immediately visible or evident (such as the depth of the rapid filter basin). It is only under these conditions 
that model limits and reality can be discussed. The modelling project can also serve as motivation for pupils to investigate a realistic system (very relevant to their education) on their own.

- Structural feasibility. In order for the modelling task to be shared among teams it is advantageous if, at the top level of the hierarchy, the system which has to be described can easily be divided up into components (themselves subsystems) which can be modelled, as far as possible, independently. The structure of a waterworks is obvious and straightforward. The raw river water is prepared in several systems located one after another - rough cleansing with the screen, the rapid filter system, the slow filter basin, sterilisation with chlorine dioxide. Each team takes over a component and prepares its own demonstration model.

- Size. In order for pupils to experience the benefits of informatics methods of modelling, the system to be modelled must be sufficiently large and consist of many components. This criterion is certainly satisfied by a waterworks. There is little sense, for example, in using object-oriented analysis and design methods such as class, object and sequence diagrams if the task is only a matter of modelling a bouncing ball.

\section{ASPECTS OF MODELLING}

\subsection{The problem of reduction}

A model is a simplified image of reality. This means that in modelling we have to decide what aspects of reality we consider are essential and which ones we wish to ignore. The reasons for reduction are primarily:

- Limited resources (development time, competence of the development team, possibilities or capabilities of development software).

- Limited knowledge of reality.

- Limited perceptive faculties of the recipients of a model.

In a school project the first point is decisive. A project can easily fail if too much is taken on at the start. This means that at the beginning of the development process the pupils have to estimate how much time is required to realise certain features. They cannot do this, of course, when they are just beginners. The problem can, for example, be checked by an evolutionary or incremental development where a minimal system is first implemented and later expanded (Sommerville 1996). 
A criterion for the selection of the elements to be presented in the model may be their significance for the whole system. The modelling of a filter process will hardly be a success without the presentation of running water! In this case the decision is based on the observation of reality.

From the aspect of media design it may be wise to consider including an unimportant aspect in the model simply because it can be visualised spectacularly. In water treatment the purification of the settling basin by a mechanical gripper is a marginal problem. On the other hand this process (in comparison to running water) looks interesting and makes the whole model worth viewing.

\subsection{Modelling as a process of media design}

At the end of the last section we mentioned that a model is also a medium for the communication of intellectual content. This means that in the design the addressee (or viewer) must be considered. Aesthetic preferences are important as are perceptive faculties and habits of thought - e.g. if the model is for pre-school age children it should not contain any written explanations. The addressee may also be the author of the model. In this case he/she invents models to make something clear to him/herself. The communication aspect is obvious in models we know of from everyday life:

- Images and models of buildings built by architects for decision-makers.

- Model-based scientific demonstrations at school.

- Didacticised explanatory models e.g. Dalton's model that explains aggregate states.

Under the perspective of communication design the following points are relevant and are discussed with respect to the waterworks example:

- Graphic design. This includes the selection of the colours and the transparency. Important parts of the model (the floating can and the gripper) should stand out optically.

- Interaction. The functioning of the fine screen can be best illustrated using an automatic simulation. For the observer, however, it is more interesting and instructive if he or she can press a button and set the machinery working.

- Selection of symbols and pictures of concrete objects. Here an empty can was chosen as an example of an object that is removed from the fine screen after being in the raw water. A floating branch or leaves would actually have been more realistic but a can is a symbol of how man pollutes water and the addressee is more shocked. 
- Design of the user interface.

- Illustration of movement. One particular problem was the visualisation of flowing water. Pupils solved this by making little bubbles float through the canal.

- Reduced illustration. An economic approach to development time is essential and results in a design problem. How can a particular motion process be illustrated as simply as possible? The mechanics of cleaning the basin in the example just consists of two moving parts.

- Exemplary processes. For the representation of a whole class of events in the real world, a single typical sequence must be selected. At a real waterworks very different objects float against the fine screen in different ways. In the animation the movement of a can was visualised to represent this.

\subsection{Modelling as an experiment}

What has been said so far throws light on the complexity and size of the problem solving processes associated with modelling. Problem solving is described by cognitive psychology as cyclical (Anderson 1996). In the search for a way from the initial state to the target the person attempting to solve the problem tries out various operators and checks whether or not they bring him closer to the target. Designers and software developers can work through parts of these cycles in their minds. Beginners do not have this experience or ability and thus need a lot of opportunities to experiment. An animated film sequence is first prepared as an experiment. In test runs the optical effect is assessed. Weak points are identified and improvements are made until the result is satisfactory.

In the waterworks project one pupil spent a whole day visualising the undulatory motion of water in a basin. He developed more than a dozen variants until he found a solution he considered acceptable. Experimenting like this develops the ability to solve problems. In a modelling project in which several persons co-operate two conditions must be met so that there is room for independent, individual experimentation.

First of all the development environment must allow pupils to prepare the model bit by bit and they must be able to test each part individually. Moreover there should be support for the rapid design of dynamic, graphic objects with no syntax errors. Macromedia Flash provides this. The definition of the course of movement of a graphic object through a "tween" usually takes seconds. Pupils can therefore go through hundreds of development cycles during a modelling project.

In the implementation of graphic software with Java using class libraries such as AWT or Swing, experimentation is more difficult and time- 
consuming. Teaching suggestions for modelling which plan an implementation in Java often assume that experimentation has finished before the first line of the program is written. Here the actual model formation is done almost exclusively with pencil and paper during the phases of analysis and design.

The second condition for individual experimentation concerns the system design. The software developed must meet the quality criterion of "changeability" to a high degree. This means that a change in one part must not have any effects on the whole system.

\subsection{Modelling as the development of a class structure}

In view of the short time available to a school project, a multimedia model is a complex, technical product that can only be managed by dividing up tasks in a team. The basis for the structuring of the development work is system design. If the object-oriented modelling paradigm is followed the program structure is represented through a system of classes (Balzert 1999). With visual software each class usually describes the features of objects visible on the monitor.

Using the UML (Unified Modelling Language) the students construct class diagrams for different parts of the Waterworks model. The classes are represented through boxes with the class names and connecting lines are used to show the relationships between the classes.

For the design of animations aggregations are very important. This concerns the relationship between an entirety (an aggregate) and its parts. For example, in one class the cleaning mechanism is defined as "Excavator". This aggregate consists of the classes "Rod" and "Grabber" in which the dynamic behaviour of the two components of the excavator are described.

In the class "Grabber", however, an object of the class "PictureGrabber" is used. This represents a static image used in the higher class "Grabber" for the definition of a film sequence. If the picture of the grabber is to be replaced later by a more realistic image, it is sufficient to just change the class "PictureGrabber". All other classes are completely unaffected.

The class "Bubble" which describes a moving (seemingly drifting in the water) air bubble only has to be described once. In the development of the model many objects (instances) can be used by a class to represent flowing water.

The division of tasks - i.e. the allocation of classes to persons who will implement them - can be done according to different criteria. One of them is the specialist knowledge of the respective pupils. Anyone with a special talent for drawing should be responsible for the design of the more difficult static images. It also makes sense if one person takes over a whole sub-tree 
of the class aggregation. In this case any later changes to the design within this area have no effect on the work of other team members.

The examples illustrate the main advantages of a good design:

- Time is saved by the multiple use of one class.

- Advance structuring of the work process.

- Achievement of changeability.

Finding a suitable class structure is a complex decision process. With visual programs it is made easier by the fact that the analysis of the reality to be illustrated already provides assistance with the structured division of the whole system. Nevertheless a class diagram of the model does not always have to be a faithful structure of the original in reality. This means the components of the software may contradict the real facts.

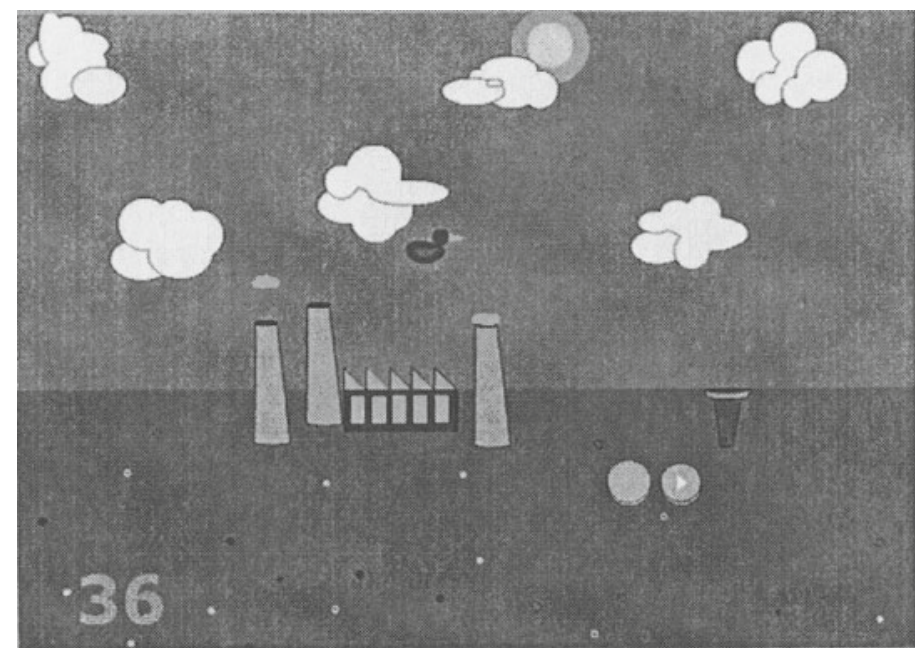

Figure 2. Erwin the duck screenshot

The following example comes from another project. In the game "Erwin the duck" a flying duck has to be guided past poisonous clouds of smoke coming from factory chimneys (Figure 2). If the duck touches a cloud it becomes unconscious and plunges to the ground.

One problem the pupils faced was the modelling of the factory chimney. They had many attempts at a class diagram and their efforts were guided by the structure of the real system. The factory is an aggregate from the building, the chimney and a dynamic cloud that keeps coming out of the chimney and is carried away by the wind. 
The problem with the implementation using Flash was that the chimney was drawn in perspective and the upper opening was represented as flat and oval. It always looked as if the cloud came out from behind the chimney not from the opening. The problem could only be resolved by breaking away from the structure of the real system and representing the chimney using two separate objects - a front and a rear. The cloud could now be positioned between them.

In this case we had to take into account the fact that Flash only supports two-dimensional graphics. In an implementation with VRML (Virtual Reality Modelling Language) the chimney-cloud problem would not have occurred. The beginning of the cloud could have been put in the interior of a three-dimensional chimney.

Note that the development of a class diagram in the framework of an object-oriented design is a creative process. It includes the facts gained from an analysis of reality and the knowledge of the possibilities of implementation. For this knowledge to be gained it is necessary for development cycles to be gone through as frequently as possible. In particular this means that modelling must not get stuck in the design phase design errors are often not obvious until after implementation.

\section{CONCLUSION}

What Karel the Robot and LOGO turtle graphics were and are for learning algorithms, tools for preparing interactive animations could be for modelling complex systems. They are platforms that support experimentation and the acquisition of knowledge through experience. In modern society animations are an important part of media culture. Their construction and reflection should therefore be part of media teaching in schools.

\section{REFERENCES}

Anderson, J. (1996) Kognitive Psychologie. Oxford, Heidelberg, Berlin.

Balzert, H. (1999) Lehrbuch der Objektmodellierung. Analyse und Entwurf. Heidelberg Berlin.

Bossel, (1987) Systemdynamik. Braunschweig.

Diener, U., Dönhoff, H-U., Rieks, K-E. and Weigend, M. (1998) Neue Medien im Unterricht Vorbild USA? (Verlag Bertelsmann Stiftung), Gütersloh.

Hubwieser, P. (1999) Modellierung in der Schulinformatik. In: LOG IN 1 1999, S.

Ossimitz, G. (2001) Didaktik der Systemdynamik, systemisches Denken. Universität Klagenfurt. 
[http://www.uni-klu.ac.at/users/gossimit/sdyn/sdyn.htm.]

Sommerville, I. (1997) Software Engineering. Addison-Wesley. 